\title{
Structures and Buildings: Referees 2018
}

The following is a list of referees who have reviewed papers for Structures and Buildings between 1 December 2017 and 30 November 2018.

The Institution of Civil Engineers is very grateful for their assistance.

We are continually looking for suitable reviewers for papers submitted to Structures and Buildings. Papers published in the Proceedings of the ICE must be submitted to at least two independent referees to judge accuracy, style, impact, importance and interest.

If you are interested in reviewing articles on any topic related to

structures and buildings, please submit your name, qualifications or CV,

\begin{tabular}{|c|c|}
\hline A Abbas & R Beale \\
\hline T Abbott & $\begin{array}{l}\text { C Bedon } \\
\text { G Behera }\end{array}$ \\
\hline M Abbu & $\begin{array}{l}\text { G Benera } \\
\text { F Bencardino }\end{array}$ \\
\hline H Abdalla & F Bernard \\
\hline J Abdalla & E Bertolesi \\
\hline S Abdel Raheem & M Betti \\
\hline A Abdelnaby & A K Bhattacharya \\
\hline F Abed & H Bilgin \\
\hline D Achillopoulou & B Binici \\
\hline J Adam & R P Borg \\
\hline A Afzali & G Boscato \\
\hline J Agarwal & S Boyd \\
\hline E Aggelopoulos & Branco \\
\hline S Agnetti & E Brunesi \\
\hline M Ahmadi & M Buitrago \\
\hline $\begin{array}{l}\text { M Ahmed } \\
\text { R Akbari }\end{array}$ & Z Bullock \\
\hline A Akbari Hamed & R Burgos \\
\hline V Akrami & C J Burgoyne \\
\hline N Al-Akhras & R S Camposinhos \\
\hline M S Alam & D Cardoso \\
\hline M Ali & M Casatont \\
\hline M Ali & C Castiglionı \\
\hline M U Ali & $\begin{array}{l}\text { M Cellikag } \\
\text { F Ceroni }\end{array}$ \\
\hline S Alizadeh & KO Cetin \\
\hline $\begin{array}{l}\text { T Alqado } \\
\text { R Alrousan }\end{array}$ & F Cetisli \\
\hline $\begin{array}{l}\text { R Alrousan } \\
\text { A Altuilåžlk }\end{array}$ & C Ch Mitropoulou \\
\hline C Amadio & R Chacon \\
\hline K M Amanat & SLChan \\
\hline J André & T M Chan \\
\hline J Andrade & W S Chang \\
\hline C Antonio & A Chen \\
\hline Y Aoki & $\begin{array}{l}\text { C Chiorean } \\
\text { K K Choi }\end{array}$ \\
\hline R Araujo & \\
\hline M Arslan & \\
\hline A Ashour & P Colombi \\
\hline F Aslani & M Constantinou \\
\hline $\begin{array}{l}\text { P Asteris } \\
\text { A Ataei }\end{array}$ & M Cordeiro Rodrigues \\
\hline $\begin{array}{l}\text { A Ataei } \\
\text { N Atmaca }\end{array}$ & M Corradi \\
\hline $\begin{array}{l}\text { N Atmaca } \\
\text { I B Valente }\end{array}$ & H Costa \\
\hline $\begin{array}{l}\text { E Båazik-Borowa } \\
\text { E Valente }\end{array}$ & L Costa Neves \\
\hline H Baghi & L Cunningham \\
\hline R Bailey & E da Nobrega Tavares \\
\hline J Balogh & C Dagenais \\
\hline M Bather & M D'Aniello \\
\hline M Bavan & H Darama \\
\hline A Beal & A Darby \\
\hline
\end{tabular}

and areas of expertise. We are in need of individuals who will agree to review papers in a timely fashion (within 3 to 4 weeks of receipt) and provide confidential feedback to the Editorial Advisory Panel concerning the quality of the paper and any suggested revisions that would be appropriate.

If you are such a person, please contact Kirsten Buchanan (tel.: +44 (0)207 665 2204; e-mail: kirsten.buchanan@icepublishing.com) for more information on the referee process.

$\begin{array}{ll}\text { D D'Ayala } & \text { J A Henriques } \\ \text { S de Andrade } & \text { B Hobbs } \\ \text { H De Backer } & \text { A Hojatkashani } \\ \text { F De Luca } & \text { A Horr } \\ \text { G De Matteis } & \text { S A Hosseini } \\ \text { A De Silva } & \text { Z Huang } \\ \text { A Del Savio } & \text { T Ibell } \\ \text { D Dellatore } & \text { A N T Ihaddoudene } \\ \text { A Dey } & \text { M Ismail } \\ \text { R Dimitri } & \text { J P Jaspart } \\ \text { J Dow } & \text { G Jones } \\ \text { Y Du } & \text { S Jones } \\ \text { A Duarte } & \text { R Judge } \\ \text { C Dundar } & \text { S Kaewunruen } \\ \text { R Durand } & \text { C C Kai } \\ \text { P Ebadi } & \text { B Kamrani Moghaddam } \\ \text { U Ebead } & \text { C Karayannis } \\ \text { T El Maaddawy } & \text { B Kasal } \\ \text { S E D El Metwally } & \text { M Kashani } \\ \text { B El-Ariss } & \text { S Kasinos } \\ \text { M Eliasova } & \text { A Kaveh } \\ \text { S El-Refaie } & \text { S Kazerani } \\ \text { H Enayati } & \text { M I Khan } \\ \text { G Ercolani } & \text { C King } \\ \text { H Erdogan } & \text { B Kromoser } \\ \text { R C Erenoglu } & \text { A Kwan } \\ \text { B Evirgen } & \text { N Lam } \\ \text { H Fang } & \text { A Lampropoulos } \\ \text { A Faroughi } & \text { N Largos } \\ \text { M Fernández Ruiz } & \text { M Latour } \\ \text { V Fernández-Dávila } & \text { D Lau } \\ \text { J Forth } & \text { M Leblouba } \\ \text { M Fouad } & \text { C Lee } \\ \text { F Fu } & \text { C S Lee } \\ \text { K Fujii } & \text { D S H Lee } \\ \text { E Garcia-Taengua } & \text { D Lehman } \\ \text { S A Ghahari } & \text { F Li } \\ \text { M Ghahremannejad } & \text { G Li } \\ \text { O Ghasemi-Fare } & \text { W Li } \\ \text { B Ghiassi } & \text { Q Liang } \\ \text { A Gilani } & \text { C Lima } \\ \text { I Giongo } & \text { N Lopes } \\ \text { M Gizejowski } & \text { R Maddalena } \\ \text { L Guizani } & \text { M Madhavan } \\ \text { S Guler } & \text { H Madkour } \\ \text { L Guo } & \text { H Mahir } \\ \text { M N Habeeb } & \text { M Malite } \\ \text { A Hadhood } & \text { P Malosta } \\ \text { R Haweeleh } & \\ \text { H Hemida } & \end{array}$




\begin{tabular}{ll} 
T Mara & M O'Connor \\
A Mari & D R Oliveira \\
E Martinelli & J Owen \\
J Martins & M Paknahad \\
K Megalooikonomou & F Parisi \\
S Mehanny & L Pelecanos \\
E Mele & A Penford \\
R Meli & J Pereiro-Barcelo \\
M Memari & D Petkova \\
L Miguel & K Pilakoutas \\
P Miguel & V Piluso \\
H Mohamed & D Pitilakis \\
D Mohotti & I Pop \\
S Mojtabaei & D Proske \\
A Molavi Tabrizi & M M Rafi \\
A Mordak & Y Rafiq \\
P Mullett & A Ramaswamy \\
J Munaiar Neto & N Raut \\
A Mwafy & T Ray \\
H Naderpour & J C Ribeiro \\
M Nahvinia & M Rodrigues \\
R Nascimbene & M Romero \\
D Nethercot & R Roy \\
T Nikolaidis & J Sagaseta \\
W Niu & D R Sahoo \\
E Noroozinejad Farsangi & A Sarmanho \\
E Norouzzadeh Tochaei & E Sayed-Ahmed \\
& \\
\hline &
\end{tabular}

J Schmid
I Shaaban
A Shaat
B Sharma
T Sheehan
H Shoukry
B Silva
P Simao
M Sitek
R Smith
M Sonebi
G Stavroulakis
M Su
G Sun
A Sweedan
D Tann
T Tannert
H Tekeli
A Tenchini
M Terrenzi
W Thanoon
M Theofanous
R Tomasi
C Topkaya
T Triantafillou
D Trujillo
F B Varona

R Vollum

P Wagh

G Wang

$X$ Wang

B Watkins

S Wong

P Xiang

$\mathrm{B} \times \mathrm{u}$

TXu

J B Yan

J Yang

Y F Yang

J Yeung

S Yilmaz

W Yuan

R Zanuttin

B Zhang

M Zhang

Q Zhang

B Zhao

$M$ Zhou

W Zhou

$X$ Zhou

S Zmigrodzki 